\title{
Tamoxifen-Induced Non-alcoholic Steatohepatitis Cirrhosis
}

\author{
Tae Hwan Kim', Young Bae Kim², Jae Youn Cheong' ${ }^{1}$, Sung Won Cho', Soon Sun Kim' \\ Departments of 'Gastroenterology and 'Pathology, Ajou University School of Medicine, Suwon, Korea
}

\begin{abstract}
Non-alcoholic fatty liver disease has been observed in over $30 \%$ of patients who have received tamoxifen therapy. However, tamoxifen-induced non-alcoholic steatohepatitis (NASH) cirrhosis has never been reported in Korea. A 41-year-old woman was diagnosed with invasive ductal carcinoma in the left breast. She had well-controlled type 2 diabetes mellitus, hypertension, and chronic hepatitis B. Ultrasonography showed mild fatty liver. Chronic hepatitis B had been treated with clevudine one month before the diagnosis of breast cancer. The patient was diagnosed with NASH cirrhosis 39 months after tamoxifen treatment. Careful observation for the development of NASH cirrhosis is warranted during tamoxifen therapy.
\end{abstract}

Keywords: Breast neoplasms; Chronic hepatitis B; Non-alcoholic fatty liver disease; Liver cirrhosis; Tamoxifen

\section{INTRODUCTION}

Breast cancer is the second most common female cancer in Korea. There has been a sharp increase in the incidence rates of breast cancer, from 20.9/100,000 women in 1999 to 44.7/100,000 women in 2012 [1]. Tamoxifen is a selective estrogen-receptor modulator used as adjuvant therapy for estrogen receptor-positive breast cancer [2]. Tamoxifen treatment for 5 years is associated with a notable reduction in the risk of breast cancer recurrence and mortality [3]. Well-known adverse events of tamoxifen are an increased risk of thromboembolic disease and uterine cancer [3]. However, evidence that tamoxifen treatment may also result in a $30 \%-40 \%$ increase in the risk for developing non-alcoholic fatty liver disease (NAFLD), has received scant attention [4].

NAFLD is a common benign liver disease, with estimates of the worldwide prevalence of NAFLD ranging from $6.3 \%$ to $33 \%$, with a median of $20 \%$, in the general population [5]. Typically, NAFLD is most likely to be associated with obesity and type 2 diabetes mellitus [5]. Patients with NAFLD have increased overall mortality and the liver-related mortality rate is higher in patients with non-alcoholic steatohepatitis (NASH) than in the control population [6].
Tamoxifen-induced NAFLD tends to be overlooked by physicians because of the high prevalence of fatty liver in the general population and the clinical significance of NAFLD is often underestimated. Here, we present a case report of NASH-related liver cirrhosis linked to tamoxifen therapy in a Korean patient with well-controlled chronic hepatitis B.

\section{CASE REPORT}

A 41-year-old woman was referred to Ajou University Hospital for a $2.0-\mathrm{cm}$ left breast mass with a $2.2-\mathrm{cm}$ enlarged left axillary lymph node in July 2011. One year prior to the breast cancer diagnosis, she had been diagnosed with hypertension, type 2 diabetes mellitus, and hypercholesterolemia, all of which were well controlled. She was taking the following medications: glimepiride (4 $\mathrm{mg})$, losartan potassium (50 mg), hydrochlothiazide (12.5 mg), and rosuvastatin calcium $(10 \mathrm{mg})$. She was also taking clevudine (30 mg) for chronic hepatitis B since June 3, 2011. At the start of clevudine therapy, hepatitis B e-antigen was negative, serum aspartate aminotransferase and alanine aminotransferase were 104 and $108 \mathrm{U} / \mathrm{L}$, respectively. The serum hepatitis B virus DNA level was $2.19 \times 10^{5} \mathrm{IU} / \mathrm{mL}$. Abdominal ultrasonography showed the 
presence of mild fatty liver. The initial body weight was $67 \mathrm{~kg}$ with a body mass index of $24.9 \mathrm{~kg} / \mathrm{m}^{2}$. She reported no alcohol consumption. Core-needle biopsy was performed on the breast mass and fine-needle aspiration biopsy was performed on the enlarged left axillary lymph node. The breast biopsy showed the presence of invasive ductal carcinoma, which was positive for the estrogen and progesterone receptors and negative for c-erb-B2 gene amplifica- tion. Lymph node aspiration biopsy result showed metastatic carcinoma. First, she was treated with neoadjuvant chemotherapy with 4 cycles of doxorubicin and cyclophosphamide, followed by 4 cycles of paclitaxel from June 14, 2011. After the neoadjuvant chemotherapy, she underwent surgery for breast cancer, which was left breast-wide local excision with left axillary lymph node dissection on January 9, 2012. Following the operation, she received ad-

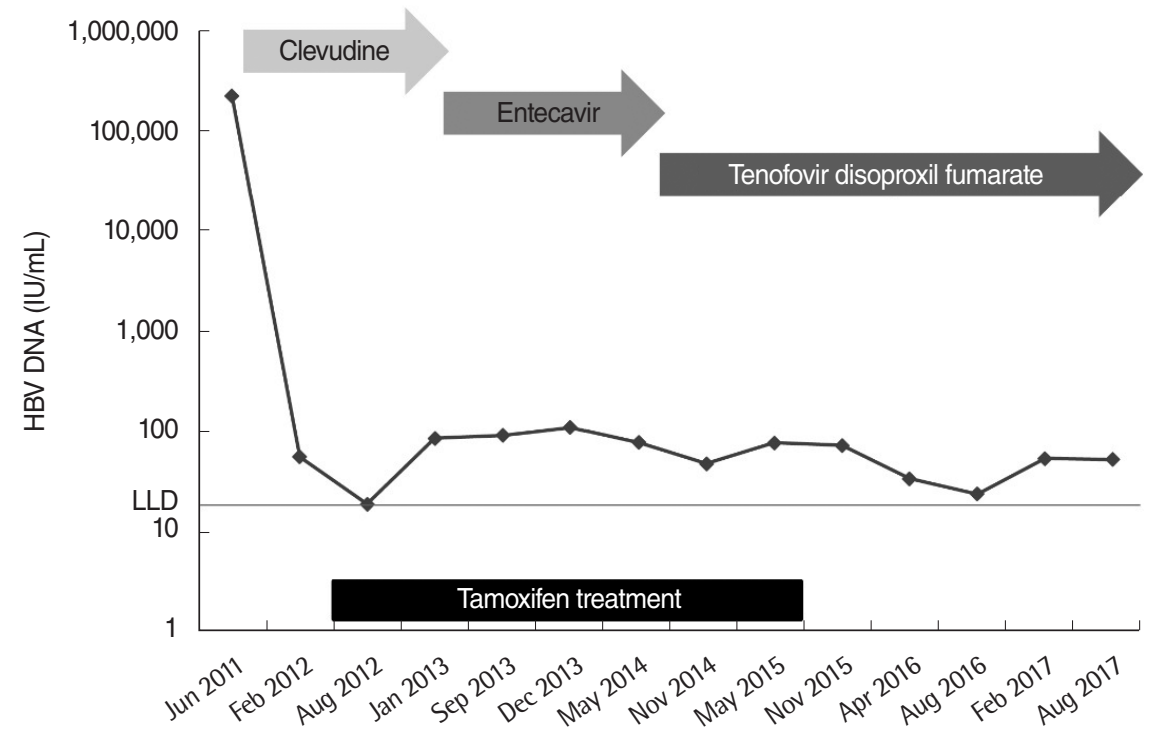

Fig. 1. Summary of chronic hepatitis B treatment. After 6 months clevudine treatment, serum HBV DNA level was controlled under $100 \mathrm{IU} / \mathrm{mL}$. HBV, hepatitis B virus; LLD, lower limit of detection.

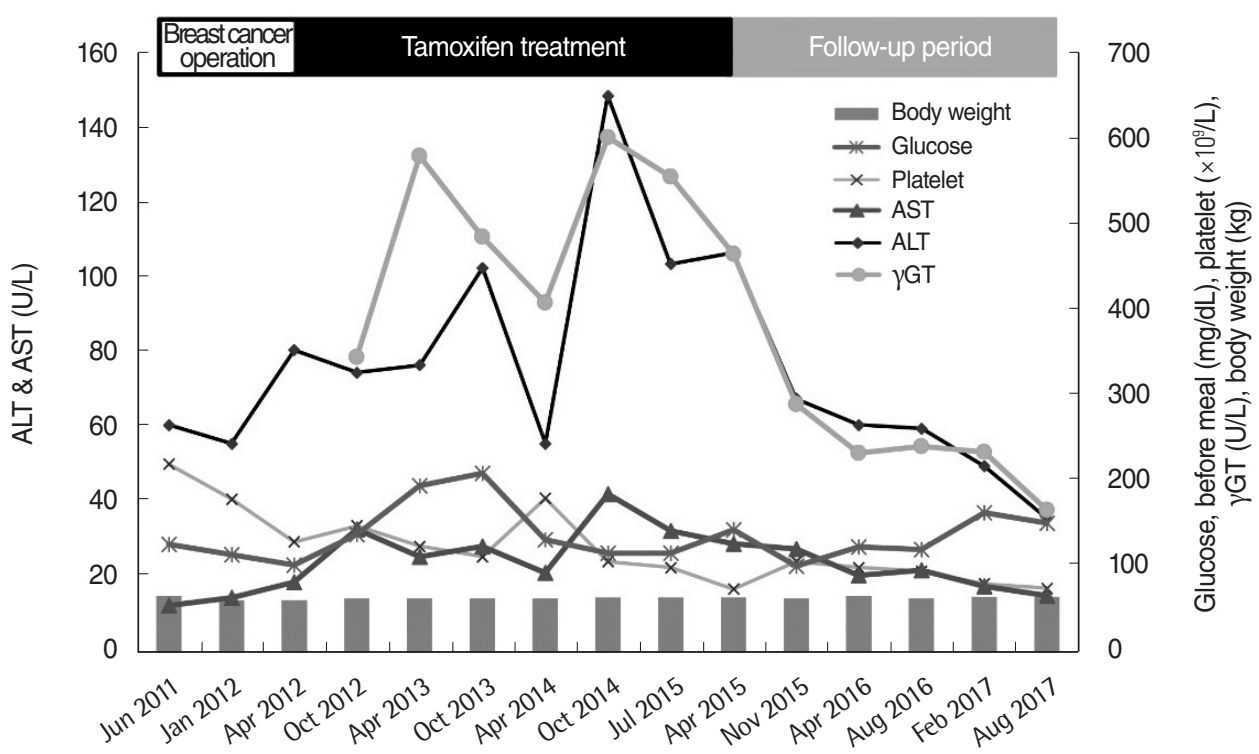

Fig. 2. Clinical course and laboratory findings. During tamoxifen therapy, ALT levels progressively increased, while platelet counts progressively decreased. Body weight and serum glucose level (before meal) showed only a minimal change during tamoxifen therapy. After stopping tamoxifen therapy, serum AST, ALT, and $\gamma \mathrm{GT}$ improved gradually; however, platelet count decreased continuously. ALT, alanine aminotransferase; AST, aspartate aminotransferase; $\gamma \mathrm{GT}$, gamma-glutamyl transpeptidase. 
juvant radiotherapy from February 2012 to March 2012. She received adjuvant hormonal therapy with tamoxifen (20 mg/day) after the radiotherapy, starting April 4, 2012. Chronic hepatitis B was controlled with clevudine until August 2012 followed by entecavir (0.5 mg) until October 2013, and subsequently, tenofovir disoproxil fumarate $(300 \mathrm{mg})$. Despite the relatively well-controlled serum hepatitis B virus DNA $(<100 \mathrm{IU} / \mathrm{mL}$ after treatment) (Fig. 1), she showed a tendency towards a decreased platelet count with elevated aspartate aminotransferase, alanine aminotransferase, alkaline phosphatase, and gamma-glutamyl transpeptidase (Fig. 2). Additional blood tests showed positive anti-nuclear antibody (1:40) and negative anti-hepatitis C virus antibody. Ul- trasonography-guided core-needle liver biopsy was performed on July 15, 2015, 39 months after tamoxifen treatment. The core of liver biopsy specimen showed small, even-sized, regenerative nodules with perisinusoidal and bridging fibrosis, and ductular proliferation forming micronodular cirrhosis (Fig. 3A). The hepatocytes revealed mild micro- and macrovesicular fatty change, and ballooning degeneration including a few Mallory bodies. There was moderate infiltration of lymphoid cells in lobule and portal to periportal areas resulting in piecemeal necrosis (Fig. 3B). Liver computed tomography showed shrinkage of liver volume with an irregular surface and splenomegaly (Fig. 4). Based on these findings, a diagnosis of liver cirrhosis due to NASH associated with
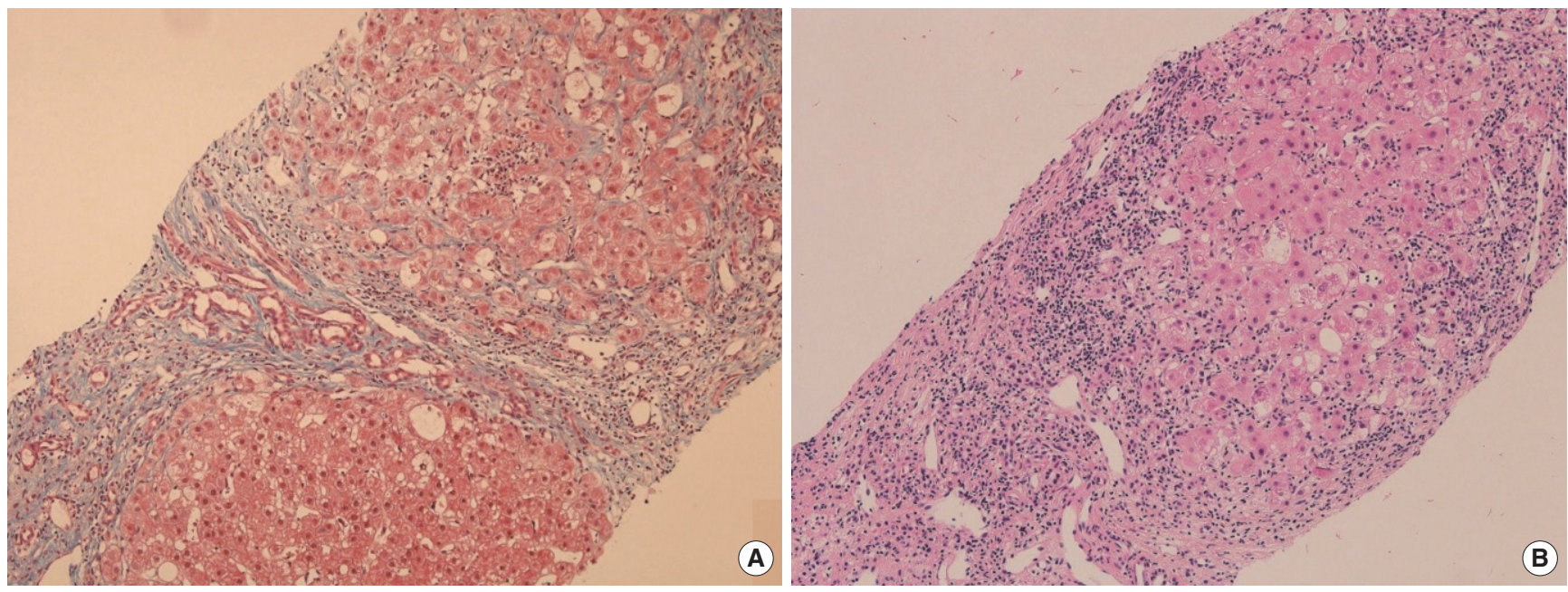

Fig. 3. Pathological findings. (A) Micronodular cirrhosis showing small, even-sized, regenerative nodules with perisinusoidal fibrosis. (B) Fatty change and degeneration including Mallory bodies, and infiltration of lymphoid cells in periportal areas.
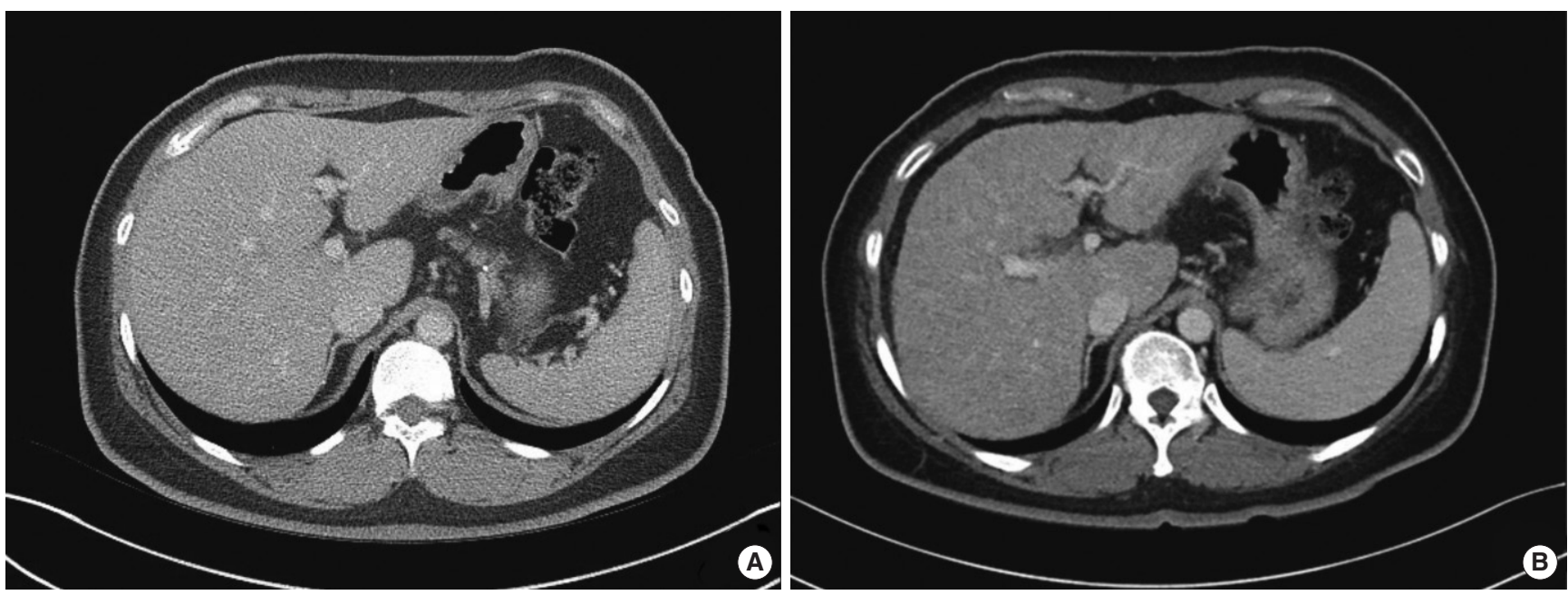

Fig. 4. Liver CT images. (A) The initial liver image showed mild fatty changes. (B) After 39 months of tamoxifen therapy, the liver CT showed shrinkage of liver volume with an irregular surface and splenomegaly. CT, computed tomography. 
tamoxifen therapy was made. Tamoxifen therapy was stopped and the patient continues to undergo close follow-up for liver cirrhosis and breast cancer. After stopping tamoxifen therapy, serum aspartate aminotransferase, alanine aminotransferase, and gammaglutamyl transpeptidase improved gradually, however, platelet count decreased continuously. Hemoglobin Alc was 7.6\% just before stopping tamoxifen and remained between $6.1 \%$ to $6.5 \%$ since discontinuing tamoxifen therapy.

\section{DISCUSSION}

To our knowledge, this is the first case report describing the development of NASH cirrhosis associated with tamoxifen use in Korea. This young female patient was diagnosed with NASH cirrhosis after treatment with tamoxifen for 3 years. Meanwhile, she had other factors, including chronic hepatitis B and type 2 diabetes mellitus, in addition to tamoxifen therapy, which could adversely influence her liver status. According to previous reports, patients receiving nucleos $(\mathrm{t})$ ide analogue therapy for hepatitis $\mathrm{B}$ usually show improved or stable fibrosis stage [7]. However, type 2 diabetes mellitus can be an important risk factor for deteriorating liver function. As discussed earlier, type 2 diabetes mellitus is a significant risk factor for NAFLD [5]. Given the rapid progression to liver cirrhosis in this case, major cause of progression to liver cirrhosis is tamoxifen and synergistically effect of type 2 diabetes mellitus also should be considered.

The association between tamoxifen and NAFLD has been reported by several studies. Tamoxifen, a selective estrogen receptor modulator, has mixed agonist and antagonist activity depending on the target tissue [2]. It was suggested that tamoxifen causes fatty liver by promoting de novo fatty acid synthesis and inhibiting fatty acid $\beta$-oxidation [8]. However, the possibility of developing NAFLD is not considered as a contraindication for tamoxifen use owing to its benign course and reversibility after drug withdrawal and a study has suggested that routine liver function testing is not required even in patients who have other risk factors for NASH [9]. However, reversibility after drug withdrawal is usually evaluated with a blood test in patients with NAFLD without cirrhosis, though data on patients with NASH cirrhosis is limited. Cases of biopsy-confirmed NASH cirrhosis after stopping tamoxifen have also been reported [10]. Therefore, regular liver function testing might be a reasonable approach to determine the optimal timing of drug withdrawal in patients who have received tamoxifen therapy. Another important point is early recognition of tamoxifen induced liver toxicity by physicians. In our case, liver biopsy was performed on the patient at a late stage even though she was followed up regularly to monitor chronic hepatitis $\mathrm{B}$.

In conclusion, this is the first reported case of the development of NASH cirrhosis after tamoxifen treatment in a Korean patient. Regular monitoring for liver function and the early notification of deterioration of liver function are warranted in patients who have received tamoxifen therapy. Other risk factors for development NAFLD should be also controlled adequately to prevent the progression of liver disease.

\section{REFERENCES}

1. Jung KW, Won YJ, Kong HJ, Oh CM, Cho H, Lee DH, et al. Cancer statistics in Korea: incidence, mortality, survival, and prevalence in 2012. Cancer Res Treat 2015;47:127-41.

2. Osborne CK. Tamoxifen in the treatment of breast cancer. N Engl J Med 1998;339:1609-18.

3. Early Breast Cancer Trialists' Collaborative Group (EBCTCG), Davies C, Godwin J, Gray R, Clarke M, Cutter D, et al. Relevance of breast cancer hormone receptors and other factors to the efficacy of adjuvant tamoxifen: patient-level meta-analysis of randomised trials. Lancet 2011;378: 771-84.

4. Akhondi-Meybodi M, Mortazavy-Zadah MR, Hashemian Z, Moaiedi M. Incidence and risk factors for non-alcoholic steatohepatitis in females treated with tamoxifen for breast cancer. Arab J Gastroenterol 2011;12: 34-6.

5. Vernon G, Baranova A, Younossi ZM. Systematic review: the epidemiology and natural history of non-alcoholic fatty liver disease and non-alcoholic steatohepatitis in adults. Aliment Pharmacol Ther 2011;34:274-85.

6. Dunn W, Xu R, Wingard DL, Rogers C, Angulo P, Younossi ZM, et al. Suspected nonalcoholic fatty liver disease and mortality risk in a population-based cohort study. Am J Gastroenterol 2008;103:2263-71.

7. Tsai NC, Marcellin P, Buti M, Washington MK, Lee SS, Chan S, et al. Viral suppression and cirrhosis regression with tenofovir disoproxil fumarate in Asians with chronic hepatitis B. Dig Dis Sci 2015;60:260-8.

8. Begriche K, Massart J, Robin MA, Borgne-Sanchez A, Fromenty B. Druginduced toxicity on mitochondria and lipid metabolism: mechanistic diversity and deleterious consequences for the liver. J Hepatol 2011;54:77394.

9. Saphner T, Triest-Robertson S, Li H, Holzman P. The association of nonalcoholic steatohepatitis and tamoxifen in patients with breast cancer. Cancer 2009;115:3189-95.

10. Oien KA, Moffat D, Curry GW, Dickson J, Habeshaw T, Mills PR, et al. Cirrhosis with steatohepatitis after adjuvant tamoxifen. Lancet 1999;353: 36-7. 